\title{
Verantwortungsvoller Umgang mit der Diagnose Schizophrenie
}

Das Ansehen der Psychiatrie hat in den letzten Monaten Schaden genommen. Kollegen haben in ihrem Gutachten den 32-jährigen rechtsradikalen Anders Breivik, der am 22. Juli 2011 unfassbare 77 überwiegend gleichaltrige und politisch interessierte Norwegerinnen und Norweger geplant und effizient ermordete, als schizophren diagnostiziert. Öffentlich widersprachen andere norwegische und internationale Psychiater. Da in den Medienberichten und Auszügen aus den Gutachten weder Ich-Störungen (Gedankenlautwerden, Gedankeneingebung, Gedankenentzug, Gedankenausbreitung), noch kommentierende oder dialogisierende Stimmen beschrieben wurden, ist anzunehmen, dass ein Wahn des Massenmörders als anhaltend, kulturell unangemessen und völlig unrealistisch - oder wie die amerikanischen Diagnosekriterien sagen „bizarr“ - eingeschätzt wurde, wodurch die Diagnose einer Schizophrenie nach beiden modernen Diagnosesystemen ICD und DSM-IV gegeben werden konnte. Angehörige der Opfer vermuten, dass der Massenmörder eine Schizophrenie simuliert hätte. Ich glaube, dass dies bei den unscharfen Grenzen unserer Diagnosen gar nicht notwendig gewesen wäre.

Es kann diese Diagnose des „Patienten Anders Breivik“ ohne genauere Kenntnis der Explorationen und Außenanamnesen weder bestätigt noch verworfen werden. Wir lesen, dass das psychiatrische Gesundheitspersonal der Haftanstalt den Mörder aufgrund seines Verhaltens im Alltag der Anstalt für normal hält. Das beunruhigende an dieser Diagnose beim Massenmörder ist, dass die Diagnose „Schizophrenie“ so leicht gegeben werden kann und sich damit auch tausende wirklich schizophrene (überwiegend ungefährliche) Patienten in einem Topf mit dem Massenmörder wiederfinden. Dies ist für die mit ihrer Schizophrenie und den Vorurteilen gegenüber Schizophrenen kämpfenden Betroffenen eine große Last. Die Allgemeinheit ist froh, sich mit dem Vorliegen einer Krankheit nicht mehr weiter mit dem schrecklichen Vorfall auseinandersetzen zu müssen. Auch der Verdacht, dass durch die begutachtenden norwegischen Psychiater mittels der Diagnose Schizophrenie das „Entlassen“ des Massenmörders nach abgesessener Haftstrafe verhindert werden soll, ist für das Ansehen der Psychiatrie als medizinische Disziplin gefährlich. Wir wollen diagnostizieren und behandeln und nicht als Spezialisten für Freiheitsentzug wahrgenommen und instrumentalisiert werden. Auch kann es nicht unsere Aufgabe sein, das kollektive Straf-, Rache- oder Schutzbedürfnis der Gesellschaft zu befriedigen.

Verschiedene Psychiatrien in Österreich berichten stark unterschiedliche Häufigkeiten an Schizophreniediagnosen und man erlebt immer wieder, dass an verschiedenen Stationen einer Klinik ein und derselbe psychiatrische Patient von verschiedenen Psychiatern einmal als Schizophrenie, das andere Mal als bipolare Störung, als schizo-affektive Störung, als wahnhafte Störung, als akutpolymorph-psychotische Störung oder auch als Persönlichkeitsstörung entlassen wird. Viele diagnostische Feinheiten aus dem Fachgebiet der Psychopathologie sind durch die modernen internationalisierten Konsensuskriterien, die in demokratischen Abstimmungen und nicht durch wissenschaftliche Untersuchungen gefunden wurden, verloren gegangen.

Die Verunsicherung der breiten Öffentlichkeit durch die oft widersprüchlichen Expertenmeinungen bei Diagnose psychiatrischer Krankheitsbilder sollte alle in der Psychiatrie Tätigen zu erhöhten Bemühungen anspornen, neue diagnostische und klassifikatorische Wege zu suchen. Wir behandeln in der Psychiatrie häufig sehr erfolgreich, die psychiatrische Diagnostik hinkt hinterher. Das schwächt die Stellung der Psychiatrie in unserer Medizin und in unserer Gesellschaft.

Peter Fischer 